\title{
Sequential consumer choice as multi-cued retrieval
}

\author{
Adam N. Hornsby, ${ }^{1,2, *}$ Bradley C. Love, ${ }^{2,3}$ \\ ${ }^{1}$ dunnhumby, 184 Shepherds Bush Road, London W6 7NL \\ ${ }^{2}$ Department of Experimental Psychology, University College London, London WC1H 0AP \\ ${ }^{3}$ The Alan Turing Institute, United Kingdom
}

*To whom correspondence should be addressed; E-mail: adam.hornsby.10@ucl.ac.uk

Whether adding songs to a playlist or groceries during an online shop, how do we decide what to choose next? We develop a model that predicts such openended, sequential choices using a process of cued retrieval from long-term memory. Using the past choice to cue subsequent retrievals, this model predicts the sequential purchases and response times of nearly 5 million grocery purchases made by over 100,000 online shoppers. Products can be associated in different ways, like by their episodic association or semantic overlap, and we find that consumers query multiple forms of associative knowledge when retrieving options. Attending to certain knowledge sources — as estimated by our model - predicts important retrieval errors, like the propensity to forget or add unwanted products. Our results demonstrate how basic memory retrieval mechanisms shape choices in real-world, goal-directed tasks. 


\section{Introduction}

Many studies of preferential choice have examined how people choose between a fixed menu of options (1-3). Yet, in real-world tasks like online grocery shopping, the space of possible options is too large to be considered at once. Choices therefore depend on how options are retrieved from long-term memory (4-7). The task itself can provide the context to retrieve associated choice options. Here, we consider how a previous choice influences subsequent consumer choices in a goal-directed sequential decision task. Using computational models, we evaluate how different sources of knowledge influence choice, by decomposing associative memory into its constituent components.

Once items are retrieved from memory, they may cue subsequent retrievals, leading to complex sequential dynamics like semantic clustering. For example, when asked to name as many animals as possible, sequential retrievals tend to be semantically similar and faster when they are so (e.g., dog $\rightarrow$ cat) (8-12). This sequential cuing of memory means that retrievals tend to cluster over time. Sequential consumer choices may also semantically cluster if they depend on the same retrieval mechanisms; we test this hypothesis here.

Retrieval is said to depend on the strength of associations in memory, although association is somewhat nebulous given that items can relate in different ways. For example, choosing tea could trigger childhood memories of enjoying it with cake, as it did for Proust (13). Options that occur in the same episode could have a high probability of being retrieved; this was shown in the early experiments of memory (14) and has since become a core prediction in models of memory search (15).

Sequential choices could also be influenced by semantic similarity between items, such as their conceptual overlap. For example, whilst they may not be consumed in the same episode, purchasing chocolate could prompt the search for other chocolate bars, due to their shared 
features. Semantic space models have been shown to predict sequential retrievals in fluency tasks $(11,16)$ and options generated to open-ended questions (7). Online shoppers may similarly retrieve products that are nearby in conceptual space when making sequential choices, given that they are not constrained by the physical layout of products in stores.

An often-cited feature of semantic memory is that people are sensitive to hierarchical relations between items. For example, responses tend to be slower when judging the correctness of statements like "apples are fruit" compared to "apples are produce" (17). One might therefore expect online shoppers to retrieve items that are nearby within a structured hierarchy, such as purchasing fruit then vegetables. This seems particularly likely during grocery shopping, given that stores tend to arrange products taxonomically in order to make them easier to locate (questions concerning whether hierarchical, semantic, and episodic knowledge are strictly separate systems from neurobiological or computational perspectives is orthogonal to our aims and claims).

We hypothesise that retrieval of options in sequential choice tasks depends on their similarity with the prior choice across different knowledge formats (visualised in Figure 1c). We test this by developing associative representations of these knowledge sources (as in (20)), before evaluating whether sequential choices are better explained by one or a combination of these representations. We also hypothesise that individual differences may drive shoppers to attend to certain sources of knowledge more than others. For example, a shopper driven by episodic memories of breakfast might retrieve butter then bread, whereas those relying on hierarchical knowledge may retrieve butter with other dairy products, as they would in the supermarket. We operationalise these processes of associative retrieval and attention in a computational cognitive model and show that it can predict sequential consumer choices. After each retrieval, we suggest that consumers accept or reject possibilities according to their goals. For example, shoppers may consider whether retrieved options are suitable for breakfast. However, goals are not modelled 


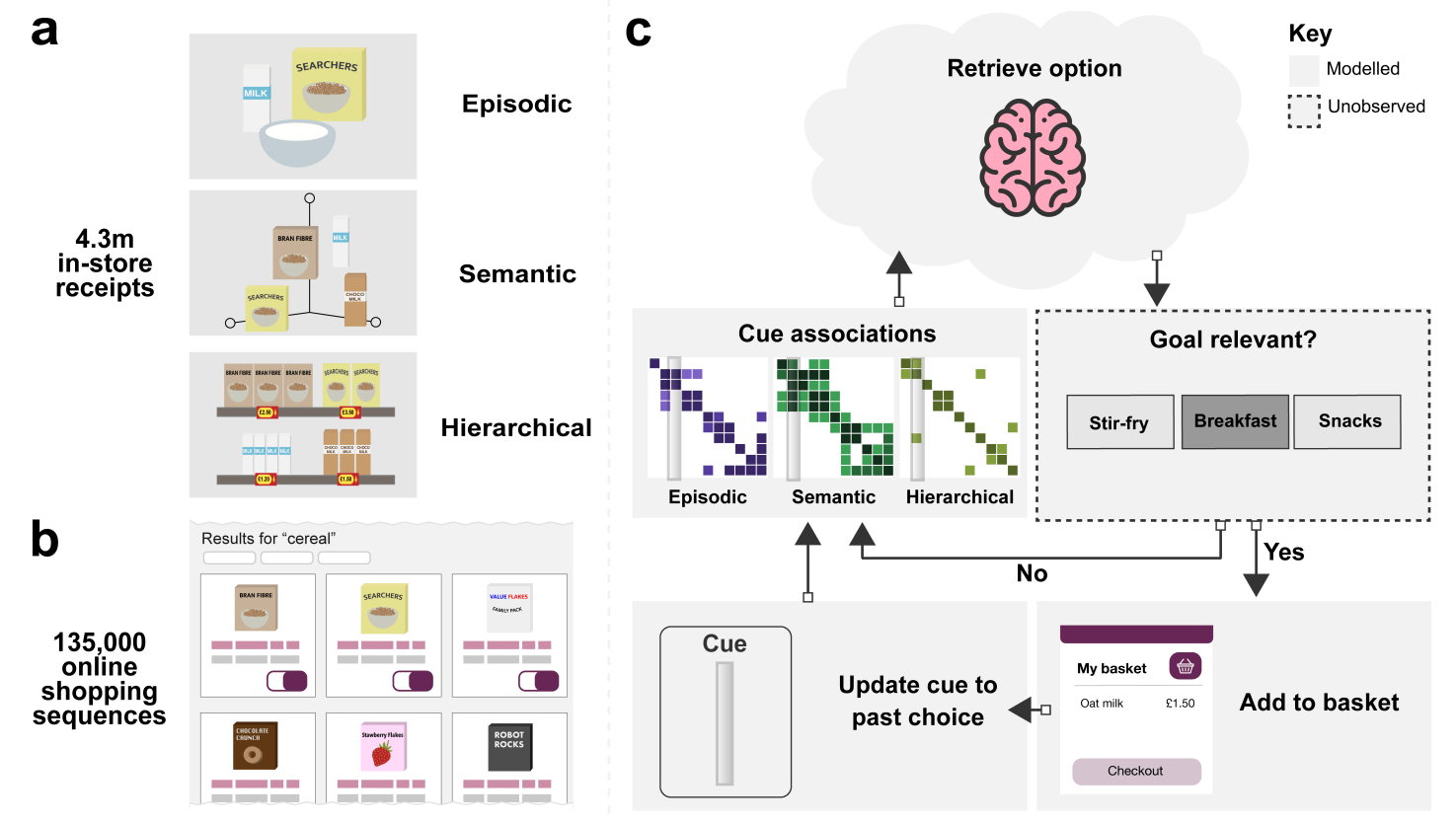

Figure 1: Deciding what to choose next when shopping for groceries online depends on cued retrieval from multiple knowledge sources. a., We used $4.3 \mathrm{~m}$ unordered, in-store receipts to build representations of episodic, semantic and hierarchical knowledge. b., To model retrieval, we collected data from 135,000 shoppers as they sequentially searched for products on the website of one of the UK's largest supermarket retailers. c., Prior choices predict future ones, by virtue of their similarity according to different representational formats. Once an item is added to their basket, shoppers use this to cue matches from long-term memory. The stronger the match with this cue, the higher the probability an item will be retrieved (this may be attenuated by increased attention towards a particular representation). Retrieved items are checked against one's internal goals. If the retrieval is goal-relevant, the shopper adds an appropriate item from the website and uses that item to cue associations. If not, a new option is retrieved and checked for goal relevance until one is accepted. Similar heuristic strategies have been used in models of option generation for single choices $(18,19)$. Once all goals are satisfied, the user checks out. Note that the goal-checking process is not modelled here. 
or enumerated here, as we focus on the contribution of different knowledge systems during sequential option retrieval.

During online grocery shopping, consumers could feasibly search for products in any order. Yet, if options are retrieved according to their similarity with the prior choice, purchases should be non-random and predicted by their sequential similarity. We test this using a new dataset of over 5 million consumer choices. To foreshadow, results supported this hypothesis. Interestingly, representations of episodic, semantic and hierarchical knowledge explained unique variance when predicting sequential choices and their response times, supporting the idea that shoppers query multiple sources of long-term knowledge. Consumers retrieving options from episodic memory appeared less prone to subsequently forget products, whereas those attending to semantic knowledge were less likely to add items to their basket that they didn't otherwise need. Thus, individuals may recruit these systems to different extents, which may affect their ability to complete the task effectively.

\section{Results}

We analyzed a dataset of sequential consumer purchases gathered from one of the UK's largest supermarket retailers. The data contained 5,238,469 choices from 132,146 unique visitors across 42,837 unique products (more information in Materials and Methods).

Shoppers were required to search sequentially for groceries to add to their virtual basket (the website is depicted in Figure S1). On average, they made 39.64 choices $(95 \% C I=$ $[39.49,39.79])$. The landing page displayed a generic selection of "special offers" (e.g., discounted products), which was used relatively infrequently to purchase products $\left(\mu_{o f f e r s}=\right.$ $1.55,95 \% C I=[1.53,1.58])$. Shoppers tended to search for products using a search bar, which was located at the top of every page $\left(\mu_{\text {searches }}=23.36,95 \% C I=[23.26,23.46]\right)$. They could also use a category drop-down by hovering the mouse over a hyperlink saying "Groceries" at 
the top of every page. This menu required users to navigate to the lowest level of three subcategories before viewing products (e.g., Cupboard $\rightarrow$ Cereals $\rightarrow$ Healthy cereals). It was used relatively infrequently $\left(\mu_{\text {category }}=3.02,95 \% C I=[2.98,3.05]\right)$. After navigating with the search bar or the category drop-down, shoppers would be shown a list of associated products, where they could purchase products or click on products for more information. Before checkout, they could also add products from a personalised recommender system that suggested products that might have been forgotten before checkout $\left(\mu_{\text {forgotten }}=0.322,95 \% C I=[0.3199,0.327]\right)$. Visitors removed an average of 3.23 products from their basket before checking out $(95 \% C I=$ $[3.20,3.27])$.

To explain sequential transitions between choices, we developed representations of episodic, semantic and hierarchical knowledge. A representation of episodic memory was derived by calculating the probability of two products co-occurring in the same basket, using a separate dataset of in-store transactions in which products appear unordered. With the same dataset, we trained a distributed representation of semantic memory using word2vec (21) (similar to the one developed in (20)). A representation of hierarchical knowledge was calculated using the retailer's five-level product taxonomy, which groups products from small subgroups (e.g., apples) to increasingly large departments (e.g., produce). Uniquely, this hierarchy describes a taxonomy of is-of relations, which we used to define a measure of similarity as opposed to distance in a continuous semantic space (see Section 1.2 of the supplemental for more information).

\section{Past choices cue subsequent retrievals}

If shoppers cue retrievals from a given long-term store, then one would expect the similarity between consecutive purchases to be higher than when compared with random permutations, where the order of products has been randomly permuted within each shopping trip. For example, given that butter and bread are episodically linked (e.g., purchased in the same baskets), this 


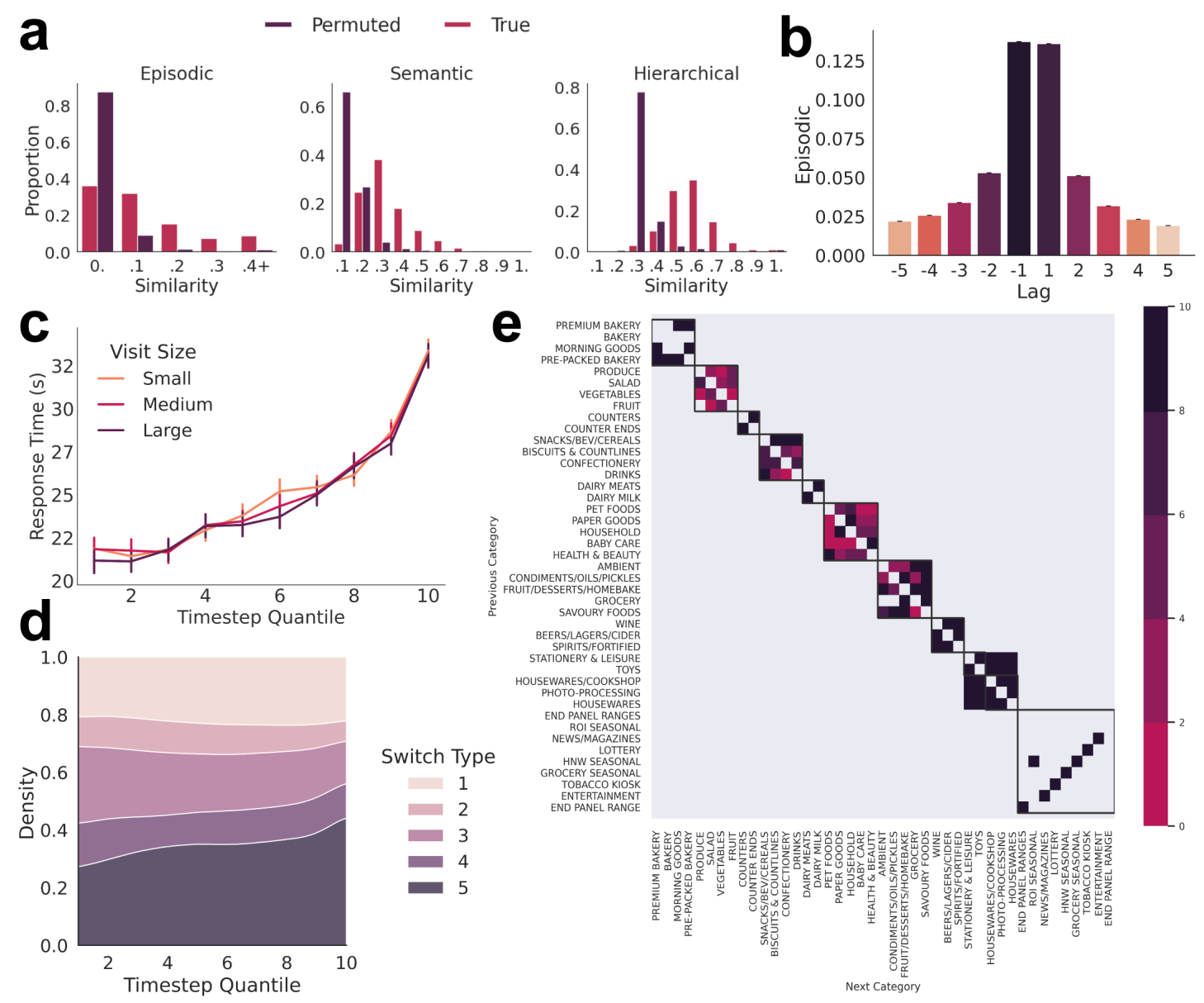

Figure 2: Consecutive purchases tend to be close episodic, semantic and hierarchical relations. a., Choices are predicted by their similarity with the prior choice across each representation. Histograms show that the similarity between consecutive purchases (averaged for each visit) was higher compared to when the order of purchases was randomly permuted. (with $95 \%$ confidence intervals). b., Sequential retrieval is like a ripple in semantic memory. Mean episodic similarity (with 95\% confidence intervals) between the current product and those purchased recently is higher compared with products purchased later c., Visitors slowed as they approached the end of their shopping trip. Mean response times (with 95\% confidence intervals) as a function of timestep quantile (Small $=10-30$ items, Medium $=31-49$ items, Large $=50+$ items)) . d., Consumers make more between-category transitions (i.e., taxonomy level five) towards the end of their visit. Stacked density plots denoting the proportion of switches according to each level of the taxonomy as a function of the relative timestep. e., Transitions between product groups at the fourth level of the hierarchy clustered into intuitive higher-order groupings that appear similar to those in the product taxonomy, suggesting that the taxonomy closely resembles how shoppers represent products during sequential choice. The Lift -1 of each transition is depicted in purple, with values less than 0 shown in grey. Boxes represent clusters identified by the optimal spectral clustering solution (more information in section 1.7. and 2.6 of the supplement). 
should increase the probability of them being chosen consecutively. As shown in Figure 2a, the average trip-wise similarity between consecutively purchased items was significantly higher for the true order of purchases compared to the permuted order for episodic $\left(\right.$ Median $_{\text {true }}=$ $0.0756, I Q R_{\text {true }}=0.1465 \&$ Median $\left._{\text {permuted }}=0.0154, I Q R_{\text {permuted }}=0.0193\right)$ (MannWhitney $\left.\mathrm{U}=2.95 \times 10^{11}, p<.0001, C L E=0.8312\right)$, semantic $\left(\right.$ Median $_{\text {true }}=0.2507$, $I Q R_{\text {true }}=0.1495 \&$ Median $\left._{\text {permuted }}=0.0770, I Q R_{\text {permuted }}=0.0669\right)($ Mann-Whitney $\mathrm{U}=$ $\left.1.5 \times 10^{11}, p<.0001, C L E=0.9141\right)$ and hierarchical representations $\left(\right.$ Median $_{\text {true }}=0.5169$, $I Q R_{\text {true }}=0.1433 \&$ Median $\left._{\text {permuted }}=0.2620, I Q R_{\text {permuted }}=0.0504\right)($ Mann-Whitney $\mathrm{U}=$ $\left.1.07 \times 10^{11}, p<.0001, C L E=0.9390\right)$. This suggests that choices were nonrandom and were cued by their similarity with the prior choice.

Sequential cued-retrieval can be viewed as a ripple through memory, in that more recently retrieved items tend to be more similar (11). As shown in Figure 2b, choices were most similar to the prior choice according to their episodic similarity (Figure S2 shows similar patterns for semantic and hierarchical knowledge). To confirm this, we calculated the average similarity of each choice for lags ranging from -10 to -1 . Regressing lag onto the standardised average similarity for each user revealed a positive average relationship across each representation, indicating that more recent choices tend to be more similar $\left(\mu_{\text {episodic }}=0.135795 \% C I=\right.$ $[0.1331,0.1383], Z_{\text {sign }}=56229.0, p<.0001, \mu_{\text {semantic }}=0.1837,95 \% C I=[0.1816,0.1859]$, $Z_{\text {sign }}=59170.5, p<.0001, \mu_{\text {hierarchical }}=0.2263,95 \% C I=[0.2247,0.228], Z_{\text {sign }}=$ $61651.5, p<.0001)$.

The retrieval model described in Figure 1 selects options according to the similarity with the previous option. As this process repeats, the chance that a high similarity option has already been purchased increases, meaning that choices should become more dissimilar over time. Grouping choices into deciles based on their timestep (and thus adjusting for different trip sizes), we regressed each similarity measure onto timestep decile and included dummy coded 
representations of each transition type as confounding variables within each regression. Results showed that average similarity between sequential choices decreased over time across episodic $\left(b_{\text {episodic }}=-0.058,95 \% C I[-0.061,-0.055], p<.0001\right)$, semantic $\left(b_{\text {semantic }}=-0.010\right.$, $95 \% C I[0.013,-0.007], p<.0001)$ and hierarchical representations $\left(b_{\text {hierarchy }}=-0.247\right.$, $95 \% C I[-0.250,-0.243] . p<.0001$ ) (full regression equations in Table S3-S5). The increase in hierarchical similarity over time is visualised in Figure 2d.

One might correspondingly expect choices to become slower over time, as more dissimilar options are slower to retrieve. Regression analyses of inter-response intervals (IRIs) conformed to this expectation. Firstly, the standardised coefficients for episodic $\left(b_{\text {episodic }}=-0.137,95 \% \mathrm{CI}\right.$ $=[-0.138-0.136], \mathrm{p}<.0001)$, semantic $\left(b_{\text {semantic }}=-0.086,95 \% \mathrm{CI}=[-0.087-0.085], \mathrm{p}<.0001\right)$ and hierarchical knowledge $\left(b_{\text {hierarchy }}=-0.273,95 \% \mathrm{CI}=[-0.274-0.271], \mathrm{p}<0.0001\right)$ were all negative predictors of IRI, indicating that more dissimilar options were slower to retrieve. Moreover, average IRIs appeared to slow over the duration of the trip $\left(b_{\text {timestep }}=0.130\right.$, $95 \% C I[0.129,0.130], p<.0001)$. This slow-down is shown in Figure 2c. Including variables representing the navigation method (e.g., keyword search) and the similarity across each representation confirmed this slow-down as a general trend (full regression equation in Table S2) and this echoes similar patterns of slowing observed in category fluency tasks (10-12).

\section{Episodic, semantic and hierarchical knowledge jointly explain sequential choice}

We next evaluated whether consumers' sequential choices were best explained by one or multiple sources of knowledge, using the retrieval equation of a popular memory retrieval model, Search of Associative Memory (SAM) (22). This equation formalizes how options may be retrieved based on their similarity with the current cue (illustrated in Figure 1c, with full equation in Section 1.3 of the supplement). Importantly, we evaluated its fit when including representa- 
Table 1: The \% BIC improvement over the random baseline and the mean attention weights (with 95\% confidence intervals) for each of the candidate models. Results show that including representations of multiple knowledge formats provides the best fit to the data (shown in bold)

\begin{tabular}{lrlll}
\hline & $\Delta$ BIC $(\%)$ & Episodic & Semantic & Hierarchy \\
\hline Episodic & 9.13 & $0.29(0.001)$ & \multicolumn{2}{l}{} \\
Semantic & 4.80 & $0.091(0.001)$ & \\
Hierarchy & 26.28 & & $2.217(0.016)$ \\
\hline Episodic \& Semantic & 12.30 & $0.258(0.001)$ & $0.068(0.001)$ & \\
Semantic \& Hierarchy & 29.10 & $0.055(0.001)$ & $2.105(0.017)$ \\
Episodic \& Hierarchy & 31.79 & $0.174(0.001)$ & $2.004(0.017)$ \\
\hline Multiple & $\mathbf{3 3 . 7 8}$ & $0.160(0.001)$ & $0.044(0.001)$ & $1.939(0.018)$ \\
\hline
\end{tabular}

tions of episodic, semantic and hierarchical knowledge. Results are presented as the mean improvement in the Bayesian Information Criterion (BIC) relative to a random model, for which the probability of each transition was equal across all remaining products (see Section 1.3 of the supplemental for further details about the model fitting procedure).

As shown in Table 1, the best fitting model contained multiple memory representations, even after penalising for multiple parameters. This suggests that online grocery shoppers query multiple knowledge formats when deciding what to choose next. A model parameter recovery study revealed that each parameter could be recovered accurately, with correlations between actual and estimated parameters $>0.6$ in all cases (reported in Section 2.8 of the supplemental). This indicates that parameter estimates were uniquely identifiable and could therefore be interpreted.

Inspecting the average attention weights of the best fitting model, one can gauge the relative importance of each representation. Hierarchical knowledge received the largest weight, followed by episodic, then semantic knowledge. Further analyses (presented in Section 2.6 of the supplemental), revealed that transitions between product groups tended to overlap with superordinate classifications in the product taxonomy (clusters of transitions between products groups 
at the third taxonomic level are visualised in Figure 2e). Together, this suggests that shoppers rely heavily on hierarchical knowledge about how products relate, which aligns closely with the taxonomy used to arrange products in stores.

We next evaluated whether response times were best explained by one or a combination of knowledge sources. A multiple linear regression was performed, predicting the IRIs between each choice, using each of the three similarity measures as predictors. We also included the number of products remaining to be purchased and dummy coded variables representing each of the navigation methods (e.g., keyword search); these served as confounding variables (full model equation in Table S6). Model comparisons that penalised for more variables revealed that IRIs were best explained by this full model, rather than one containing a subset of similarity measures (model comparisons in Table S7).

Importantly, these results support our key claim that sequential choice in open-ended tasks depends on retrieval of options from multiple sources of long-term memory. It is perhaps surprising that episodic and semantic knowledge explain unique variance in consumer choices, given that the latter may derive from the former (23). However, episodic knowledge provides a more direct link between experiences than semantic knowledge, which may play a unique role during goal-directed choice. Most of all, shoppers appeared to depend on hierarchical knowledge about products, which emphasises the influence of taxonomic organisations during navigation of large option spaces.

These model fits demonstrate the complementary role of different knowledge systems during everyday sequential choice tasks, but should not be limited to such settings. For example, they should extend to more well-known experimental tasks, such as semantic fluency. To test this, we fit the same retrieval model to a separate dataset of sequential food retrievals collected in a controlled experiment (originally collected by (24) and shared via (25)). In this task, 50 participants were given three minutes to retrieve as many food words as possible. Much like keyword 
searches, each retrieval was typed into a text box. For each word retrieved (e.g., "hamburger"), we found a corresponding product from the retailer, allowing us to measure the episodic, semantic and hierarchical similarity between sequential retrievals as before (more details of the method and results can be found in Section 3 of the supplemental). After performing the same set of model comparisons, results showed that the best fitting model contained all three representations, even after penalising for the additional parameters. Moreover - much like shoppers

— participants appeared to rely most on hierarchical knowledge when sequentially retrieving food items from memory. This suggests that these knowledge systems also influence sequential retrievals in controlled experimental tasks and that our model fits are representative of memory retrieval and not merely the design of the website.

\section{Relying on certain knowledge formats predicts retrieval errors}

If shoppers rely on certain knowledge formats during retrieval, this may increase their propensity to make certain errors such as forgetting or falsely retrieving products. Forgetting indicates the failure to retrieve a relevant item (i.e., a miss) whereas removing items indicates the failure to suppress irrelevant retrievals (i.e., a false-alarm). Indeed, forgetting is often viewed as a failure of retrieval $(26,27)$ and could simply result from "searching the wrong part of memory" (page 40, (28)).

The retrieval model used here (22) assumes that items will be activated according to a process of spreading activation $(29,30)$. When operating on an episodic representation, this would tend to chain together products found together in the same basket (e.g., purchasing a Thai pepper may cue coconut milk, bamboo shoots and other complementary ingredients). Thus, we hypothesised that shoppers relying on episodic knowledge — as measured by the attention weights from the best fitting retrieval model — would be less likely to forget products, as they would tend to co-activate items often combined in pursuit of a goal. Forgotten items were measured 

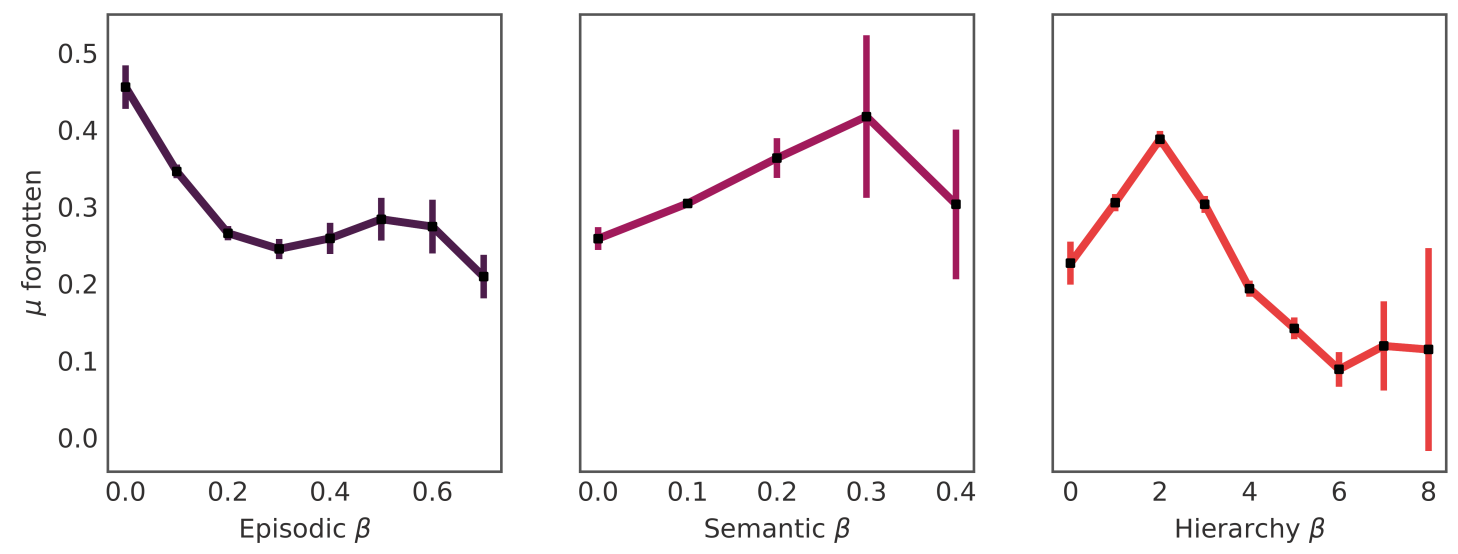

Figure 3: Mean number of forgotten items (with 95\% confidence intervals) for each model attention weight $(\beta)$. Results show that relying on episodic or hierarchical knowledge predicted fewer forgotten items, whereas attending to semantic knowledge predicted more forgotten items, as measured by use of a recommender system displayed before checkout.

through the use of recommender system, which displayed products the shopper had purchased recently and frequently in prior visits before checkout.

When operating on a semantic network, a spreading activation process would tend to coactivate products that are substitutable, or conceptually similar but not necessarily purchased together (e.g., purchasing a Thai pepper may co-activate other forms of pepper). We therefore hypothesised that shoppers relying more on semantic knowledge would be more prone to remove products from their basket, indicating that they didn't actually need them. This shares a kindred spirit with theories of confabulation in memory retrieval $(31,32)$, where high semantic similarity between studied items causes related items to be erroneously retrieved.

\section{Forgetting products}

As shown in Figure 3, results supported the prediction that shoppers with increased episodic retrieval forgot fewer items on average $\left(r_{s}=-0.0811,95 \% C I[-0.0868,-0.0754], p<\right.$ .0001). In addition, attending to hierarchical knowledge predicted fewer forgotten items $\left(r_{s}=\right.$ $-0.0088,95 \% C I[-0.0145,-0.0031], p \leq .003)$. In contrast, the more shoppers attended to 
semantic knowledge, the more likely they were to forget items $\left(r_{s}=0.0152,95 \%\right.$ CI $[0.0095$, 0.0209], $p=0.0001)$.

To gauge their relative usefulness, we scaled each attention weight and entered them into a multiple linear regression, regressing onto the number of forgotten items. We also included the number of choices and the proportion of choices made using each search context as confounding variables. The regression was significant $\left(F_{8,117328}=178.7, \mathrm{p}<0.0001, R^{2}=.012\right)$. Importantly, higher attendance to episodic knowledge $\left(b_{\text {episodic }}=-0.053,95 \% \mathrm{CI}=[-0.059\right.$, $0.048], \mathrm{p}<.0001)$ or hierarchical knowledge $\left(b_{\text {hierarchy }}=-0.041,95 \% \mathrm{CI}=[-0.046,-0.035], \mathrm{p}\right.$ $<0.0001)$ negatively predicted forgetting, whereas attending more closely to semantic knowledge $\left(b_{\text {semantic }}=0.015,95 \% \mathrm{CI}=[0.009,0.021], \mathrm{p}<=0.0001\right)$ positively predicted forgetting (full regression equation reported in Table S9).

Relying on episodic knowledge generates more complementary retrievals but could also indicate greater experience with products and their relationships. Both could explain the general trend of forgetting fewer items as one increases attendance to episodic knowledge. Moreover, the formalisation of episodic knowledge predicts that shoppers will transition to products that frequently co-occur with the past choice. Thus, another possibility is that the recommended products were less relevant to those that used episodic knowledge to guide their search, because they had been purchased by that shopper with a high frequency in the past. More work - perhaps through a cognitive battery — is required to understand the relationship between retrieval from different knowledge systems and forgetfulness.

\section{Removing products}

We next examined whether shoppers attending to certain knowledge sources removed more items from their basket. In line with our predictions, shoppers who attended more closely to the semantic similarity between items removed more items from the basket $\left(r_{s}=0.0992,95 \%\right.$ 
$C I[0.0935,0.1049], p<.0001)$. Conversely, those attending to episodic knowledge $\left(r_{s}=\right.$ $-0.014,95 \% C I[-0.0197,-0.0083], p<.0001)$ or hierarchical knowledge $\left(r_{s}=-0.084\right.$, $95 \% C I[-0.0897,-0.0783], p<.0001)$ removed fewer items on average.

As before, we regressed each of these descriptors onto the number of items removed from each basket. We also included the number of choices and the proportion of choices made using each search context as confounding variables. The regression was significant $\left(F_{8,117328}=\right.$ $\left.2202.0, p<.0001, R^{2}=.131\right)$. Increased attention to semantic similarity was shown to positively predict the number of items removed $\left(b_{\text {semantic }}=0.051,95 \% C I=[0.012,0.089], p<\right.$ $.0001)$. Conversely, higher attendance to episodic knowledge $\left(b_{\text {episodic }}=-0.359,95 \% C I=\right.$ $[-0.398,-0.319], p<.0001)$ and hierarchical knowledge $\left(b_{\text {hierarchy }}=-0.870\right.$,

$95 \% C I=[-0.909,-0.831], p<.0001)$ predicted fewer removed items (full regression equation in Table S10).

Relying on semantic knowledge generates more substitutable retrievals, which could explain why these shoppers tended to add products to their basket that they don't otherwise need. Further analyses (presented in Section 2.10.1 of the supplemental) revealed that the removed items tended to have above average similarity with the chosen options across each knowledge representation, which is supportive of this idea. Another possibility is that shoppers attending to episodic knowledge were more experienced with products and their associations and thus were less prone to mistakes. We leave these possibilities for future analyses.

Most importantly, these results provide further support for the main claim that shoppers query multiple knowledge formats when deciding what to choose next and that individuals may differ in the extent to which they rely on these systems. Future experimental work may wish to test these findings using explicit measures and thus causally evaluate the precise relationship between attention to representations and errors. Such studies would complement our claim that knowledge formats may be recruited by individuals to different extents. 


\section{Discussion}

In open-ended choice tasks like grocery shopping, how do we decide what to choose next? Many factors could influence what is chosen, but we propose that much depends on the similarity with the preceding choice across multiple knowledge formats. This view makes a number of predictions that we confirmed. First, choices and their response times were predicted by their similarity with the last choice, suggesting that choices cue the retrieval of subsequent options. Second, this behaviour was best explained by a mixture of episodic, semantic, and hierarchical knowledge, suggesting that consumers reason about associations between products in different ways by querying different sources of knowledge. Thirdly, how prone consumers were to different types of memory errors was predicted by their reliance on different types of memory, as assessed by model fits.

As our model describes, retrieved options may be cued by prior choices. This likely explains why the sequential choices of online grocery shoppers clustered over time, as they do in fluency tasks $(8,10-12,33)$. We build upon past research $(4-7,34)$ by showing how memory retrieval mechanisms influence the generation of options in sequential decision-making tasks. These results would not be predicted by many classical models of preferential choice, which consider option-retrieval to be out-of-scope (1-3). Our results demonstrate how choice options can be dynamically constructed in the moment depending on the context supplied by the previous choice. Future work could explore the influence of other past retrievals, which have been shown to influence list recall (for a review, see (15)).

Choices may follow different trajectories depending on which sources of knowledge are queried. Overall, choices and their response times were best explained by the sequential similarity across episodic, semantic, and hierarchical representations. In addition, individual differences in the extent to which each representation was recruited predicted how many products 
would be forgotten or removed. This would not be predicted by many existing models of semantic memory retrieval $(11,12)$ and option generation $(6,7)$, which rely on a single measure of association. Associative knowledge likely takes several forms (e.g., see (35)), which is consistent with our modelling approach and results. Future experimental work may wish to explore the role of different associations in memory retrieval tasks and whether such systems are cognitively or neurally distinct.

Although we focused on sequential retrieval of choice options, determining whether an option is goal relevant could also be key to choice (see Figure 1c). Whilst modelling goals was out of the scope of this study, we hope studying the interaction between goals and retrievals will be addressed in future work. A person's subjective preferences may also affect which retrieved options are chosen $(6,36,37)$. Choice itself can affect preferences (37), which in turn may affect memory retrieval. For example, new episodic memories could be formed after purchasing a preferred pairing of balsamic vinegar and bitter salad. One exciting direction for future research is to consider how different shopping experiences for individuals lead to different memory representations, which in turn affect future purchasing decisions.

One possible confound is that sequential choices were biased by the design of the website. For example, adding different brands of cola from the same page could cause retrievals to appear more hierarchical, as they belong to the same sub-category. To test this, we re-ran all analyses on a filtered dataset of product transitions that occurred through use of the search bar (detailed in Section 2.11 of the supplemental). All results were consistent with those reported here, which is reassuring given that these transitions were perhaps best representative of memory-based search. In addition, the model that best explained sequential grocery choices also provided the best fit to sequential retrievals of foods, which were observed in a controlled laboratory task (detailed in Section 3 of the supplemental). Thus, whilst design features may help shoppers to retrieve certain brands (e.g., brands of cola), shoppers still seem to depend on cued retrieval 
from multiple knowledge formats to determine what they look for next.

Analyses of large field data such as these complement findings from the lab, allowing theories of memory and cognition to be evaluated at an unprecedented scale with high ecological validity. In this case, we've shown that the sequential purchases of grocery shoppers are well explained by a model of memory retrieval that was originally developed to explain behaviour in lab tasks $(11,22)$. A large driver of this model's success in this task is that it makes use of three relevant embedding spaces that relate to knowledge systems proposed in studies of memory (38). We hope these findings stimulate further work in the lab, where one typically has a higher degree of control for assessing questions of cause and effect. For example, an additional explanation for choices becoming slower and more dissimilar over time is that retrieved options are increasingly rejected as they become less goal relevant (e.g., goals become increasingly satisfied). Future lab studies could assess this claim by asking participants to choose options in the presence of more or fewer goals. Others could enquire about the content of people's goals and examine how they interact with choices over time.

Online shoppers may be more or less responsive to certain recommendations depending on their navigational strategy. Results showed that shoppers relying on episodic memory were less likely to purchase products from a recommender system that reminded shoppers of previous purchases before checkout. This may be of practical significance to marketers designing personalised recommender systems, who could adapt recommendations to suit the retrieval strategies of shoppers as estimated by our cognitive model. For example, shoppers relying on hierarchical knowledge could benefit from recommendations promoting episodically related products (e.g., "goes well with...") whereas those relying on episodic knowledge could benefit from seeing semantically similar products (e.g., "people also viewed...") Such insights would complement traditional machine learning systems, which do not typically consider variations in human cognition (39). 
Our approach may make it possible to use shopping behavior to detect cognitive impairments. Longitudinal studies link performance in retrieval tasks to memory decline in preclinical Alzheimer's populations (40). While many people shop, relatively few people participate in such clinical tests until they experience serious memory impairment, thereby foregoing the advantages of an early diagnosis (41). Although more work would be needed to establish efficacy and suitable ethical guidelines, model fits (e.g., changes in attendance to episodic memory cues) may in the future predict the onset of cognitive impairment. Such a system operating at scale with informed consent could improve outcomes for individuals and society.

To conclude, our findings suggest that grocery shoppers use their previous choice to query associations across multiple knowledge systems when determining their next purchase. Depending on which sources of knowledge are queried, shoppers may choose products in different orders or exhibit an increased propensity to forget. Working with models and memory formats originally developed in laboratory settings, we were able to verify and extend these ideas in a real-world setting. In doing so, we strengthen the case for the complementary nature of laboratory and large-scale, real-world studies $(20,42)$ with linkages enhanced through common modelling approaches.

\section{Materials and methods}

\section{Clickstream data}

Data capturing a sequence of clicks during a given shopping session is known as clickstream data. In this study, we used clickstream data collected by a large British retailer between 1st January 2015 and 31st March 2016. We used a random sample of visits resulting in a checkout during that period, each from a different customer and only kept observations where a product was added to a shopper's basket (more information in Section 1.1 of the supplemental). By shopping online, all customers were required to participate in the loyalty scheme of the retailer 
and therefore consented to having their data used for research. In order to preserve user privacy, we removed all customer identifiers from the data and kept only a cryptographic hash of each visit ID. All analyses were in compliance with UCL's code of ethics

\section{In-store data}

To prevent information leaking into our analysis of online shopping behaviour, we used a distinct dataset of in-store shopping behaviour to develop knowledge representations. In-store grocery receipts are unordered, making it particularly useful for this study. The final dataset contained purchase information from 4,336,917 distinct baskets. We followed the same procedure of encryption as with the clickstream data in order to preserve the privacy of customers.

\section{Forgotten items}

Forgotten items were flagged through use of a personalised recommender system, which prompted users about items they may have forgotten at the end of their visit, before they checked-out. The exact products shown to each customer were determined according to the recency and frequency of purchase in previous shops (online or in-store), de-duplicated against products that had been purchased in the present visit. This page was displayed to users prior to payment.

\section{Removed items}

Shoppers could also remove products from their basket at any time during the shop. The total number of removed items were counted for each user.

Further details about the method can be found in Section 1 of the supplemental.

\section{References and Notes}

1. P. W. Glimcher, A. Rustichini, Science (New York, N.Y.) 306, 447 (2004). 
2. J. R. Busemeyer, J. Rieskamp, Handbook of choice modelling (Edward Elgar Publishing, 2014).

3. A. Rangel, C. Camerer, P. R. Montague, Nature Reviews Neuroscience 9, 545 (2008).

4. L. Keller, J. Ho, IEEE Transactions on Systems, Man, and Cybernetics 18, 715 (1988).

5. A. Kalis, S. Kaiser, A. Mojzisch, Frontiers in Psychology 4 (2013).

6. Z. Zhang, et al., Proceedings of the National Academy of Sciences 118 (2021).

7. S. Bhatia, Journal of Experimental Psychology: Learning, Memory, and Cognition 45, 627 (2019).

8. W. A. Bousfield, C. H. W. Sedgewick, The Journal of General Psychology 30, 149 (1944).

9. A. K. Troyer, M. Moscovitch, G. Winocur, neuropsychology 11, 138 (1997).

10. P. J. Gruenewald, G. R. Lockhead, Journal of Experimental Psychology: Human Learning and Memory 6, 225 (1980).

11. T. T. Hills, M. N. Jones, P. M. Todd, Psychological Review 119, 431 (2012).

12. J. T. Abbott, J. L. Austerweil, T. L. Griffiths, Psychological Review 122, 558 (2015).

13. M. Proust, In Search of Lost Time (Modern Library, New York, 1913).

14. H. Ebbinghaus, Memory: A contribution to experimental psychology, Memory: A contribution to experimental psychology (Teachers College Press, New York, NY, US, 1913).

15. M. J. Kahana, Annual Review of Psychology 71, 107 (2020).

16. M. N. Jones, D. J. K. Mewhort, Psychological Review 114, 1 (2007). 
17. A. M. Collins, M. R. Quillian, Journal of Verbal Learning \& Verbal Behavior 8, 240 (1969).

18. J. G. Johnson, M. Raab, Organizational Behavior and Human Decision Processes 91, 215 (2003).

19. G. Klein, S. Wolf, L. Militello, C. Zsambok, Organizational Behavior and Human Decision Processes 62, 63 (1995).

20. A. N. Hornsby, T. Evans, P. S. Riefer, R. Prior, B. C. Love, Computational brain \& behavior 3, 162-173 (2019).

21. T. Mikolov, I. Sutskever, K. Chen, G. Corrado, J. Dean, Proceedings of the 26th International Conference on Neural Information Processing Systems - Volume 2, NIPS'13 (Curran Associates Inc., Red Hook, NY, USA, 2013), pp. 3111-3119.

22. J. G. W. Raaijmakers, R. M. Shiffrin, Psychology of Learning and Motivation, G. H. Bower, ed. (Academic Press, 1980), vol. 14, pp. 207-262.

23. M. L. Mack, A. R. Preston, B. C. Love, Nature communications 11, 1 (2020).

24. J. C. Zemla, K. Cao, K. D. Mueller, J. L. Austerweil, Behavior Research Methods 52, 1681 (2020).

25. J. C. Zemla, K. Cao, K. D. Mueller, J. L. Austerweil, SNAFU: the Semantic Network and Fluency Utility, [Dataset] https://github.com/AusterweilLab/snafu-py accessed on $13 / 10 / 2021$ (2020).

26. R. M. Shiffrin, Science 168, 1601 (1970).

27. M. C. Anderson, R. A. Bjork, E. L. Bjork, Journal of Experimental Psychology: Learning, Memory, and Cognition 20, 1063 (1994). 
28. J. R. Bettman, Journal of Marketing 43, 37 (1979).

29. J. R. Anderson, Journal of Verbal Learning and Verbal Behavior 22, 261 (1983).

30. A. M. Collins, E. F. Loftus, Psychological Review 82, 407 (1975). Place: US Publisher: American Psychological Association.

31. J. Deese, Journal of Experimental Psychology 58, 17 (1959).

32. H. L. Roediger, K. B. McDermott, Journal of Experimental Psychology: Learning, Memory, and Cognition 21, 803 (1995).

33. J. Avery, M. N. Jones, CogSci (2018).

34. S. Kaiser, et al., Cognitive, Affective \& Behavioral Neuroscience 13, 814 (2013).

35. D. Mirman, J.-F. Landrigan, A. E. Britt, Psychological Bulletin 143, 499 (2017).

36. D. J. Levy, P. W. Glimcher, Journal of Neuroscience 31, 14693 (2011).

37. A. N. Hornsby, B. C. Love, Cognition 200, 104244 (2020).

38. E. Tulving, American Psychologist 40, 385 (1985).

39. T. L. Griffiths, Cognition 135, 21 (2015).

40. K. D. Mueller, et al., Archives of Clinical Neuropsychology 30, 448 (2015).

41. J. Rasmussen, H. Langerman, Degenerative Neurological and Neuromuscular Disease 9, 123 (2019).

42. R. L. Goldstone, G. Lupyan, Topics in Cognitive Science 8, 548 (2016).

43. M. N. Jones, J. Willits, S. Dennis, Models of Semantic Memory (2015). 
44. R. Agrawal, T. Imieliński, A. Swami, Proceedings of the 1993 ACM SIGMOD international conference on Management of data (1993), pp. 207-216.

45. K. L. Stachenfeld, M. M. Botvinick, S. J. Gershman, Nature neuroscience 20, 1643 (2017).

46. A. Aka, S. Bhatia, Journal of Experimental Psychology: General (2021).

\section{Acknowledgements}

We thank the Editor and anonymous reviewers for their helpful feedback. We also thank Aleksandar Kolev for comments on an earlier draft of this article and David Errington for helpful discussions during the analyses. We also thank Marco Lattanzio and his team for maintaining the servers. A.N.H. is supported by Dunnhumby and the Royal Commission for the Exhibition of 1851. B.C.L. is supported by the Royal Society Wolfson Fellowship 1830295, Wellcome Trust Senior Investigator Award WT106931MA, and National Institute of Child Health and Human Development Grant 1P01HD080679.

\section{Author contributions statement}

A.N.H. and B.C.L. designed research. A.N.H. performed research and analyzed data. A.N.H. and B.C.L. wrote the paper.

\section{Additional information}

The authors declare no competing interests.

\section{Data availability}

All data needed to evaluate the conclusions in the paper are present in the paper and/or the Supplementary Materials. 
Modelling code and data will be shared upon publication at:

https://osf.io/xw8zk/

\section{Supplementary materials}

- Further details about Materials and Methods

- Supplementary results

- Figures S1 and S2

- Tables S1 to S21 